\title{
Specialization of Zero Cycles ${ }^{\dagger}$
}

\author{
By \\ János KOLLÁR*
}

Let $X$ be a proper scheme over a field $K$. There are two ways of organizing the points of $X$ into equivalence classes using rational curves. One is the notion of $R$-equivalence introduced by [Manin72]. Two points $x_{1}, x_{2} \in X(K)$ are called directly R-equivalent if there is a morphism $p: \mathbb{P}^{1} \rightarrow X$ with $p(0: 1)=x_{1}$ and $p(1: 0)=x_{2}$. This generates an equivalence relation called $\mathrm{R}$-equivalence. The set of R-equivalence classes $X(K) / R$ forms a set.

Closely related to it is rational equivalence. Here we allow pairs of morphisms $h: C \rightarrow \mathbb{P}^{1}$ and $p: C \rightarrow X$ and declare $p_{*}\left(h^{-1}(0: 1)\right)$ and $p_{*}\left(h^{-1}(1: 0)\right)$ to be rationally equivalent. Rational equivalence is sometimes hard to see geometrically. The set of rational equivalence classes forms a group $\mathrm{CH}_{0}(X)$.

Let $S$ be the spectrum of a local Dedekind ring with residue field $k$ and quotient field $K$. Let $X_{S} \rightarrow$ Spec $S$ be a proper morphism, $x_{K}$ a closed point of $X_{K}$ and $x_{S}$ its closure in $X_{S}$. Then $x_{S} \cap X_{k}$ is a zero cycle on $X_{k}$. This defines a specialization map on zero cycles. It is easy to prove (see, for instance, [Fulton84, 2.3]), that this descends to specialization maps

$$
\mathrm{CH}_{0}\left(X_{K}\right) \rightarrow \mathrm{CH}_{0}\left(X_{k}\right) \text { and } X_{K}(K) / R \rightarrow X_{k}(k) / R \text {. }
$$

In general there is very little that one can say about these maps.

If $X_{S} \rightarrow$ Spec $S$ is a family of curves, then the specialization maps are neither surjective nor injective.

The only reasonable case when surjectivity holds is when $X_{S}$ is smooth over $S$ and $S$ is Henselian. If this is assumed then injectivity holds only if $X_{S} \cong \mathbb{P}_{S}^{1}$.

Communicated by S. Mori. Received July 25, 2003.

2000 Mathematics Subject Classification(s): Primary 14C15, 14G27, 14M20;

Secondary $14 \mathrm{D} 15$.

${ }^{\dagger}$ This article is an invited contribution to a special issue of Publications of RIMS commemorating the fortieth anniversary of the founding of the Research Institute for Mathematical Sciences.

*Princeton University, Princeton NJ 08544-1000

e-mail: kollar@math.princeton.edu

(C) 2004 Research Institute for Mathematical Sciences, Kyoto University. All rights reserved. 
The aim of this paper is to study higher dimensional cases where the specialization map is an isomorphism. The correct higher dimensional analogs of rational curves are the separably rationally connected or SRC varieties. See [Kollár01] for an introduction to their theory and [Kollár96, IV.3] for a more detailed treatment. There are many equivalent conditions defining this notion. The definition given below essentially says that two general points can be connected by a rational curve. In positive characteristic we also have to be mindful of some inseparability problems.

Definition 1. A smooth, proper variety $X$ is called separably rationally connected or $S R C$, if there is a variety $U$ and a morphism $F: U \times \mathbb{P}^{1} \rightarrow X$ such that the induced map

$$
F\left({ }_{-},(0: 1)\right) \times F\left(_{-},(1: 0)\right): U \rightarrow X \times X
$$

is dominant and separable.

The main result is the following:

Theorem 2. $\quad$ Let $S$ be the spectrum of a local, Henselian, Dedekind ring with residue field $k$ and quotient field $K$. Let $X_{S} \rightarrow \operatorname{Spec} S$ be a smooth proper morphism. Assume that $X_{k}$ is SRC. Then

(1) The specialization map on R-equivalence

$$
X_{K}(K) / R \rightarrow X_{k}(k) / R \quad \text { is an isomorphism of sets. }
$$

(2) If $k$ is perfect, the specialization map on the Chow group of zero cycles

$$
\mathrm{CH}_{0}\left(X_{K}\right) \rightarrow \mathrm{CH}_{0}\left(X_{k}\right) \text { is an isomorphism of groups. }
$$

If $k$ is not perfect, we will get that $\mathrm{CH}_{0}\left(X_{K}\right) \rightarrow \mathrm{CH}_{0}\left(X_{k}\right)$ is an isomorphism modulo $p$-torsion where $p=$ char $k$. I do not know any example where we do not have an isomorphism.

The second part of (2) has been known in case $k$ is finite. The surface case is in [Colliot-Thélène83]. For cubic hypersurfaces it is done in [Madore03], the general case is treated in [Kollár-Szabó03]. When $k$ is finite, both sides are trivial. The first part has been known in case $k$ is finite and sufficiently large (depending on the dimension and degree of $X$ under some projective embedding), see [Kollár-Szabó03]. In all of these cases the idea is to prove that the triviality of the Chow group or of R-equivalence over a finite field is shown by some "nice" maps $p: \mathbb{P}_{k}^{1} \rightarrow X_{k}$, and these can be lifted to $X_{K}$.

In general, we have to deal with the lifting of arbitrary maps $\mathbb{P}_{k}^{1} \rightarrow X_{k}$. This is accomplished using an observation of [Gr-Ha-St03, 2.4]. 
A theory for lifting of families of torsors over finite group schemes was developed in the papers [Moret-Bailly01, Moret-Bailly03]. Roughly speaking, his method implies (2.1) for varieties of the form $\mathbb{A}^{n} / G$, without assuming the existence of a smooth compactification.

As an application of the method of the proof of (2) we obtain two further results. The first is an upper semi continuity statement for the number of R-equivalence classes in families of varieties over local fields (20):

Theorem 3. Let $K$ be a local field and $f: X \rightarrow Y$ a smooth, projective $K$-morphism whose fibers are SRC. Then

$$
Y(K) \ni y \mapsto\left|X_{y}(K) / R\right| \quad \text { is upper semi continuous }
$$

in the p-adic topology (20).

Remark 4. It is quite likely that $y \mapsto\left|X_{y}(K) / R\right|$ is actually a continuous function (that is, locally constant). All varieties with a given reduction form an open set, thus the continuity follows from (2) if every fiber $X_{y}$ has a smooth SRC reduction.

It is also likely that the analogously defined function $y \mapsto\left|\mathrm{CH}_{0}^{0}\left(X_{y}\right)\right|$ is also continuous.

The second application asserts that R-equivalence and direct $\mathrm{R}$-equivalence coincide over large fields (22). The precise assertion (23) is actually much stronger.

The third part of the paper uses specialization to singular varieties to get two types of examples of quartic hypersurfaces:

Example 5. For every $n \geq 5$ there is a smooth quartic hypersurface $H^{n} \subset \mathbb{P}^{n+1}$ over $\mathbb{Q}(t)$ such that $H^{n}$ is unirational (over $\mathbb{Q}(t)$ ) and it has infinitely many R-equivalence classes.

The same example also has infinitely many R-equivalence classes over the Laurent series field $\mathbb{Q}((t))$ and even over its real closure.

By a theorem of Springer, if a smooth quadric hypersurface defined over $K$ has a point in an odd degree extension of $K$, then it has a point in $K$ itself. Similarly, it was conjectured by Cassels and Swinnerton-Dyer that if a smooth cubic hypersurface of dimension $\geq 2$ over $K$ has a point in an extension of $K$ whose degree is not divisible by 3 , then it has a point in $K$ itself. Many cases of this have been proved in [Coray76].

The next example shows that no similar result holds for quartics. 
Example 6. For every odd $d$ and $n \gg 1$ there is a smooth quartic $H_{d}^{n} \subset \mathbb{P}^{n+1}$ over $K=\mathbb{Q}(t)$ such that $H_{d}^{n}$ has a point in a field extension of degree $d$ but it does not have a point in any field extension of smaller odd degree.

\section{$\S 1$. Deformations of Combs}

Let $X_{S} \rightarrow$ Spec $S$ be a smooth morphism as in (2) and $x_{K}, y_{K} \in X_{K}(K)$ points with specializations $x_{k}, y_{k} \in X_{k}(k)$. Let $C_{k} \subset X_{k}$ be a rational curve showing that $x_{k}, y_{k}$ are R-equivalent. (2.1) follows if every such $C_{k}$ can be lifted to a rational curve $C_{S} \subset X_{S}$ passing through $x_{K}, y_{K}$. This is a deformation theory problem with known obstructions in the first cohomology of the normal bundle of $C_{k}$ twisted by $\mathcal{O}_{C_{k}}\left(-x_{k}-y_{k}\right)$ (if $C_{k}$ is smooth). We run into difficulties if this first cohomology group is not zero.

Following the method of [Ko-Mi-Mo92], we try to deal with this problem by attaching auxiliary curves $A_{i}$ to $C_{k}$ and deform the resulting reducible curve $C_{k}^{*}:=C_{k} \cup A_{1} \cup \cdots \cup A_{m}$.

It was observed in [Ko-Mi-Mo92] that for suitable $A_{i}$ and large $m$ the deformation theory of $C_{k}^{*} \subset X$ is better behaved than the deformation theory of $C_{k}$ itself. [Ko-Mi-Mo92] concentrated on the deformations of the morphism $C_{k}^{*} \rightarrow X$, in which case the obstructions never vanish.

Recently, [Gr-Ha-St03] proved that if we look at a suitable Hilbert scheme instead, then obstructions vanish for general choices of the $A_{i}$. For arithmetic applications this is a crucial improvement.

Definition 7. Let $C$ be a geometrically reduced projective curve over a field $k$. A comb over $C$ with $n$-teeth is a reduced projective curve $C \cup A_{1} \cup \cdots \cup$ $A_{n}$ having $n$ more irreducible components over $\bar{k}$. $C$ is called the handle. The other $n$ components, $A_{1}, \ldots, A_{n}$ are smooth rational curves, disjoint from each other and intersect $C$ transversally in $n$ distinct smooth points. The curves $A_{1}, \ldots, A_{n}$ may not be individually defined over $k$. A comb can be pictured as below:

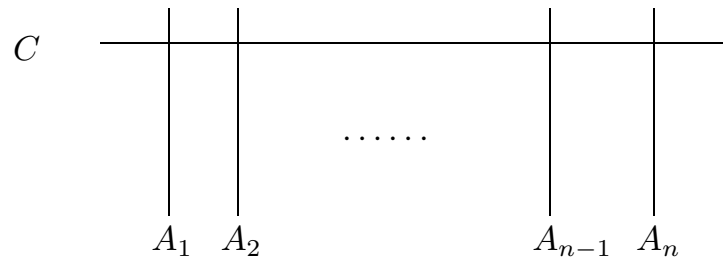

Comb with $n$-teeth 
8 (Construction of combs). Let $X$ be a smooth projective variety over an algebraically closed field $\bar{k}$. Let $C \subset X$ be a reduced local complete intersection curve with ideal sheaf $I_{C}$. (In our applications, $C$ will have only nodes.) Let $N_{C}:=\operatorname{Hom}\left(I_{C} / I_{C}^{2}, \mathcal{O}_{C}\right)$ denote the normal bundle.

The tangent space of the Hilbert scheme of $X$ at $[C]$ is $H^{0}\left(C, N_{C}\right)$ and the obstructions lie in $H^{1}\left(C, N_{C}\right)$. Our aim is to create a comb $C^{*}$ with handle $C$ such that $H^{1}\left(C^{*}, N_{C^{*}}\right)$.

To do this, assume that we have a collection of smooth rational curves $\left\{A_{w}: w \in W\right\}$ such that the following 3 conditions are satisfied:

(1) The normal bundle $N_{A_{w}}$ is semi positive for every $w$. (That is, it is a direct sum of line bundles of nonnegative degrees.)

(2) Every $A_{w}$ intersects $C$ in a single point, and the intersection is transverse. Let this point be $p=p_{w}, L_{p, w} \subset T_{p, X}$ the tangent line of $A_{w}$ at $p$ and $\bar{L}_{p, w}$ its image in the fiber $N_{p, C}$ of $N_{C}$ over $p$.

(3) There is a dense set of smooth points $p \in C$ such that the lines

$$
\bar{L}_{p, w}: p \in A_{w} \text { span } N_{p, C} \text { (as a vector space). }
$$

Remark 9. For an algebraically closed field, the collection of smooth rational curves $\left\{A_{w}: w \in W\right\}$ can be chosen to be an algebraic family. In the applications to nonclosed fields, however, the collection $\left\{A_{w}: w \in W\right\}$ will not be an algebraic family, rather a subset of an algebraic family determined by certain arithmetic conditions, see (13).

In practice, the first condition is usually easy to satisfy. If $F: W \times \mathbb{P}^{1} \rightarrow X$ is a dominant and separable morphism then $F^{*} T_{X}$ is semi positive on the general $\mathbb{P}_{w}^{1}$ and so is the relative normal sheaf $F^{*} T_{X} / T_{\mathbb{P}^{1}}$. If $F$ is an embedding on the general $\mathbb{P}_{w}^{1}$, we can pass to an open set $W^{0} \subset W$ to ensure that (8.1) holds.

(8.2) is also easy to achieve if $\operatorname{dim} X \geq 3$, see [Kollár96, II.3.]. We need rational curves through a point with ample normal bundle. These exist an any SRC variety.

The key condition is (8.3). This can be satisfied in two important cases.

First, if $X$ is SRC. Indeed, in this case for every $p \in X$ and every tangent direction $v \in T_{p} X$ there is a rational curve $f: \mathbb{P}^{1} \rightarrow X$ such that $f(0: 1)=p$ and it has tangent direction $v$ there (cf. [Kollár98, 5.2]).

Second, assume that there is a morphism $h: X \rightarrow B$ to a smooth curve $B$ whose general fibers are SRC. Let $C \subset X$ be a curve such that $h: C \rightarrow B$ is 
separable. At a general point $p \in C$ the fiber of the normal bundle $N_{p, C}$ can be identified with $T_{p} X_{p}$ where $X_{p}$ denotes the fiber through $p$. The SRC case now gives enough rational curves in $X_{p}$ to satisfy (3). This is the case used in [Gr-Ha-St03].

Assume now that we have $X, C$ and the curves $\left\{A_{w}: w \in W\right\}$ as in (8.1-3). Pick $w_{i} \in W, i=1, \ldots, m$ such that the $A_{w_{i}}$ intersect $C$ in distinct smooth points. Then the union

$$
C^{*}=C\left(w_{1}, \ldots, w_{m}\right):=C \cup A_{w_{1}} \cup \cdots \cup A_{w_{m}}
$$

is a reduced curve which is a local complete intersection along $C$.

Lemma 10 [Gr-Ha-St03]. Let $X$ be a smooth projective variety, $C \subset$ $X$ a geometrically reduced local complete intersection curve and $\left\{A_{w}: w \in W\right\}$ a collection of curves satisfying the conditions (8.1-3). Let $M$ be any line bundle on $C$.

Then there are $w_{1}, \ldots, w_{m} \in W$ such that the resulting curve $C^{*}=$ $C\left(w_{1}, \ldots, w_{m}\right)$ satisfies the following three conditions:

(1) $\left.N_{C^{*}}\right|_{C}$ is generated by global sections.

(2) $H^{1}\left(C,\left.M \otimes N_{C^{*}}\right|_{C}\right)=0$.

(3) The above two conditions hold for any other $C\left(w_{1}, \ldots, w_{m}, \ldots, w_{m+n}\right)$ obtained by adding more curves to $C^{*}$.

Proof. By Serre duality,

$$
H^{1}\left(C,\left.M \otimes N_{C^{*}}\right|_{C}\right) \quad \text { is dual to } \operatorname{Hom}\left(M \otimes \omega_{C}^{-1}, I_{C^{*}} /\left.I_{C^{*}}^{2}\right|_{C}\right) .
$$

The key point is to understand how $I_{C^{*}} /\left.I_{C^{*}}^{2}\right|_{C}$ changes if we add a new curve $A_{w_{m+1}}$ with intersection point $p=p_{m+1}$. Set $C^{*}\left(w_{m+1}\right)=C\left(w_{1}, \ldots, w_{m+1}\right)$. Let $L(p) \subset N_{p, C}$ be the tangent line of $A_{w_{m+1}}$ with dual map $q_{p}: N_{p, C}^{*} \rightarrow L(p)^{*}$. Let further $r_{p}: I_{C^{*}} /\left.I_{C^{*}}^{2}\right|_{C} \rightarrow N_{p, C}^{*}$ denote the restriction. We have an exact sequence

$$
0 \rightarrow I_{C^{*}\left(w_{m+1}\right)} /\left.I_{C^{*}\left(w_{m+1}\right)}^{2}\right|_{C} \stackrel{r_{p}}{\rightarrow} I_{C^{*}} /\left.I_{C^{*}}^{2}\right|_{C} \stackrel{q_{p} \circ r_{p}}{\longrightarrow} L(p)^{*} \rightarrow 0 .
$$

Pick any

$$
\phi \in \operatorname{Hom}\left(M \otimes \omega_{C}^{-1}, I_{C^{*}} /\left.I_{C^{*}}^{2}\right|_{C}\right) .
$$

There is an open set of points $p \in C$ such that $\phi$ has rank one at $p$. Thus the composition

$$
r_{p} \circ \phi: M \otimes \omega_{C}^{-1} \otimes k(p) \rightarrow N_{p, C}^{*}
$$


is an injection. By assumption (8.3), there is a curve $A_{w_{m+1}}$ such that the induced map

$$
q_{p} \circ r_{p} \circ \phi: M \otimes \omega_{C}^{-1} \otimes k(p) \rightarrow N_{p, C}^{*} \rightarrow L(p)^{*}
$$

is nonzero. Thus

$$
\phi \notin \operatorname{Hom}\left(M \otimes \omega_{C}^{-1}, I_{C^{*}\left(w_{m+1}\right)} /\left.I_{C^{*}\left(w_{m+1}\right)}^{2}\right|_{C}\right) .
$$

Hence by adding suitable curves $A_{w_{s}}$ we eventually achieve (10.2) and the vanishing still holds by adding more points.

Global generation of $\left.N_{C^{*}}\right|_{C}$ can be guaranteed by the vanishing of $H^{1}(C$, $\left.\left.\mathcal{O}_{C}(-P) \otimes N_{C^{*}}\right|_{C}\right)$ for all $P$, which in turn follows from the vanishing of the single cohomology group $H^{1}\left(C,\left.L^{-1} \otimes N_{C^{*}}\right|_{C}\right)$ where $L$ is any line bundle of degree $\geq 2 g(C)$.

11 (Dealing with extra intersections). In all of our cases one can arrange that the $A_{w_{i}}$ are disjoint from each other. Then $C^{*}$ is a comb in $X$ and this is what we want.

In general we may have two curves, say $A_{i}$ and $A_{j}$, intersecting at a point $x \in X \backslash C$. One can get around such extra intersections with one of the following tricks.

First, we can replace $X$ with a smooth but nonseparated scheme $X^{\prime}$ where the point $x$ is replaced by 2 points $x_{i}$ and $x_{j}$. We can lift $A_{i}$ to $A_{i}^{\prime}$ going through $x_{i}$ and $A_{j}$ to $A_{j}^{\prime}$ going through $x_{j}$. This way we remove an intersection point. The local deformation theory of the Hilbert scheme is the same on nonseparated schemes by [Artin69], and this is all we need. The global theory is more problematic but we do not use it.

Second, we can replace $X$ with $X^{\prime}=X \times \mathbb{P}^{1}$. We then replace the curves $A_{w}$ by graphs of suitable isomorphisms in $A_{w} \times \mathbb{P}^{1}$. We also have to add the trivial rational curve $\{p\} \times \mathbb{P}^{1}$ to our collection $\left\{A_{w}\right\}$.

If $X$ itself is of the form $Y \times \mathbb{P}^{n}$ for some $n \geq 1$ then we can move curves in $X$ itself, so a further product with $\mathbb{P}^{1}$ is not needed.

Both of these methods are suitable for our current purposes.

Proposition 12 [Gr-Ha-St03]. Let $X$ be a smooth projective variety, $C \subset X$ a geometrically reduced local complete intersection curve and $\left\{A_{w}: w \in\right.$ $W\}$ a collection of curves satisfying the conditions (8.1-3). Let $M$ be any line bundle on $C$.

Then there are $w_{1}, \ldots, w_{m} \in W$ such that (after possibly passing to a scheme $X^{\prime}$ as in (11)) the resulting comb $C^{*}=C\left(w_{1}, \ldots, w_{m}\right)$ satisfies the following three conditions: 
(1) $N_{C^{*}}$ is generated by global sections.

(2) $H^{1}\left(C^{*}, M^{*} \otimes N_{C^{*}}\right)=0$, where $M^{*}$ is the unique extension of $M$ to $C^{*}$ which has degree 0 on the other components.

(3) The above two conditions hold for any other $C\left(w_{1}, \ldots, w_{m}, \ldots, w_{m+n}\right)$ obtained by adding more curves to $C^{*}$.

Proof. Everything follows from (10) and the exact sequences

$$
\left.\left.0 \rightarrow \sum_{i} N_{C^{*}}\right|_{A_{w_{i}}} \otimes \mathcal{O}\left(-p_{i}\right) \rightarrow N_{C^{*}} \rightarrow N_{C^{*}}\right|_{C} \rightarrow 0
$$

and

$$
\left.0 \rightarrow N_{A_{w_{i}}} \rightarrow N_{C^{*}}\right|_{A_{w_{i}}} \rightarrow k \rightarrow 0
$$

13 (Nonclosed fields). In our applications we work over a field $k$ which is not algebraically closed and we want to obtain a curve $C^{*}$ which is defined over $k$. Assume that we have a solution $C^{*}=C\left(w_{1}, \ldots, w_{m}\right)$ of (10) over $\bar{k}$. Let $A_{w_{1}}, \ldots, A_{w_{s}}$ denote all the conjugates of the curves $A_{w_{1}}, \ldots, A_{w_{m}}$. Then $C^{* *}:=C\left(w_{1}, \ldots, w_{s}\right)$ is defined over $k$. Moreover, (10) applies if the curves $A_{w_{1}}, \ldots, A_{w_{s}}$ intersect $C$ in distinct points.

This is a somewhat troublesome condition which is arithmetic in nature. The problem is essentially the following:

Given $C \subset X$, find points $p \in C$ and rational curves $p \in A \subset X$ defined over $k(p)$. We also want $k(p) / k$ to be separable.

It is clearly enough to consider this problem for irreducible curves.

Let $C$ be an irreducible curve, $C_{g e n} \in C$ the generic point and $v_{1}, \ldots, v_{n}$ a basis of the fiber of $N_{C}$ over $C_{g e n}$. Let $H\left(C_{g e n}, v_{i}\right)$ be an irreducible component of the Hilbert scheme of smooth rational curves in $X$ with ample normal bundle intersecting $C$ only at $C_{g e n}$ with tangent direction $v_{i}$. Each $H\left(C_{g e n}, v_{i}\right)$ is a smooth variety over $k\left(C_{g e n}\right)=k(C)$ which can be extended to a $k$-variety

$$
\tau_{i}: H\left(C, v_{i}\right) \rightarrow C
$$

which parametrizes smooth rational curves in $X$ with ample normal bundle intersecting $C$ in a single point and whose tangent vector at that point is the corresponding specialization of $v_{i}$. 
We need to find points $p_{j} \in C$ such that each $\tau_{i}^{-1}\left(p_{j}\right)$ has a $k\left(p_{j}\right)$-point.

Since $\tau_{i}$ is generically smooth, we can take curve sections $B_{i} \subset H\left(C, v_{i}\right)$ such that $\tau_{i}: B_{i} \rightarrow C$ is dominant and separable. Let

$$
B \subset B_{1} \times_{C} \cdots \times_{C} B_{n}
$$

be an irreducible component with projection $\tau: B \rightarrow C$.

Lemma 14. Let $k$ be a field and $f: B \rightarrow C$ a dominant morphism of smooth curves over $k$. Then there is a dense set of points $q_{i} \in B$ such that $k\left(q_{i}\right)=k\left(f\left(q_{i}\right)\right)$ and $k\left(q_{i}\right) / k$ is separable.

Proof. Pick a point $c \in C$ such that $f$ is finite over $c$. Let $h: f^{-1}(c) \rightarrow \mathbb{A}^{1}$ be an embedding and extend it to a separable rational function $h$ which is not constant on any geometric irreducible component of $B .(f, h)$ gives a morphism $C \rightarrow C^{\prime} \subset B \times \mathbb{P}^{1}$ which is birational onto its image.

Let $u \in \mathbb{P}^{1}$ be any point such that the second projection $\pi_{2}: C^{\prime} \rightarrow \mathbb{P}^{1}$ is smooth over $u$. Then any $q \in \pi_{2}^{-1}(u)$ has the required property.

Putting everything together, we obtain the following results about the existence of "good" combs over arbitrary fields.

Theorem 15. Let $X$ be a smooth, proper, SRC variety over a field $k$. Let $C$ be a geometrically reduced local complete intersection curve, $S \subset C$ a finite set of smooth points. Let $g: C \rightarrow X$ be a morphism and $G: C \hookrightarrow X \times \mathbb{P}^{n}$ an embedding lifting $g$.

Then there is a comb $C^{*} \subset X \times \mathbb{P}^{n}$ defined over $k$ with handle $G(C)$ such that

(1) $N_{C^{*}}$ is generated by global sections.

(2) $H^{1}\left(C^{*}, \mathcal{O}_{C^{*}}(-G(S)) \otimes N_{C^{*}}\right)=0$.

(3) $C^{*}$ is smooth at $G(S)$.

Theorem 16. Let $X$ be a smooth, proper, variety over a field $k$ and $f: X \rightarrow B$ a morphism to a smooth curve whose general fibers are SRC. Let $C$ be a geometrically reduced local complete intersection curve, $S \subset C$ a finite set of smooth points. Let $g: C \rightarrow X$ be a morphism such that $f \circ g: C \rightarrow B$ is separable and $G: C \hookrightarrow X \times \mathbb{P}^{n}$ an embedding lifting $g$.

Then there is a comb $C^{*} \subset X \times \mathbb{P}^{n}$ defined over $k$ with handle $G(C)$ whose teeth are contained in fibers of $f$ such that 
(1) $N_{C^{*}}$ is generated by global sections.

(2) $H^{1}\left(C^{*}, \mathcal{O}_{C^{*}}(-G(S)) \otimes N_{C^{*}}\right)=0$.

(3) $C^{*}$ is smooth at $G(S)$.

\section{§. Proof of the Main Theorem}

The specialization map on zero cycles is surjective since every point in $X_{k}$ has a lifting to $X_{K}$ by the Hensel property. Thus it is enough to prove the following 2 assertions:

(1) If the specializations of 2 points are directly R-equivalent, then the 2 points are also R-equivalent, and

(2) If the specializations of 2 zero cycles are rationally equivalent, then the 2 zero cycles are also rationally equivalent.

17 (Proof of $(2.1)$ ). Let $u_{K}, v_{K} \in X_{K}(K)$ be 2 points with specializations $u_{k}, v_{k}$. If $u_{k}, v_{k}$ are directly R-equivalent, then there is a morphism $f: \mathbb{P}_{k}^{1} \rightarrow X_{k}$ such that $f(0: 1)=u_{k}$ and $f(1: 0)=v_{k}$. (If $u_{k}=v_{k}$ then we choose the constant map.)

Consider the diagonal map

$$
F: \mathbb{P}^{1} \rightarrow X \times \mathbb{P}^{1}, \quad p \mapsto(f(p), p) .
$$

Its image is a smooth rational curve $C \subset X \times \mathbb{P}^{1}$ connecting $u_{k}^{\prime}:=\left(u_{k},(0: 1)\right)$ and $v_{k}^{\prime}:=\left(v_{k},(1: 0)\right)$.

By (15) there is a comb $C^{*}$ defined over $k$ such that $C^{*}$ is smooth at $u_{k}^{\prime}, v_{k}^{\prime}$ and $H^{1}\left(C^{*}, \mathcal{O}_{C^{*}}\left(-u_{k}^{\prime}-v_{k}^{\prime}\right) \otimes N_{C^{*}}\right)=0$.

Mostly for ease of reference, we replace $X \times \mathbb{P}^{1}$ by its blow up $Z_{S}:=$ $B\left(X_{S} \times \mathbb{P}^{1}\right)$ along the 2 sections $\left(u_{S},(0: 1)\right)$ and $\left(v_{S},(1: 0)\right)$. Let $E_{u}, E_{v} \subset Z$ denote the exceptional divisors. Let $H_{S} \rightarrow$ Spec $S$ denote the relative Hilbert scheme of one dimensional subschemes of $Z_{S}$.

Let $\left[C^{*}\right] \in H_{S}$ be the point corresponding to (the birational transform of) $C^{*}$. By the theory of Hilbert schemes (cf. [Kollár96, I.2.15]) $H^{1}\left(C^{*}, \mathcal{O}_{C^{*}}\left(-u_{k}-\right.\right.$ $\left.\left.v_{k}\right) \otimes N_{C^{*}}\right)=0$ implies that $H_{S} \rightarrow \operatorname{Spec} S$ is smooth at $\left[C^{*}\right]$. By the Hensel property, there is a morphism $\sigma: \operatorname{Spec} S \rightarrow H_{S}$ such that $\sigma(\operatorname{Spec} k)=\left[C^{*}\right]$. Thus $\sigma(\operatorname{Spec} K)$ corresponds to a reduced genus zero curve $C_{K}$, defined over $K$ which has intersection number 1 with $E_{u}$ and $E_{v}$. This implies that $C_{K} \cap E_{u}$ and $C_{K} \cap E_{v}$ are $K$-points of $C_{K}$. The projection of $C_{K}$ to $X_{K}$ therefore shows that $u_{K}$ and $v_{K}$ are directly R-equivalent. 
18 (Proof of $(2.2))$. This is done in 2 steps.

(1) First we prove that if 2 zero cycles $Z_{K}^{1}, Z_{K}^{2} \in X_{K}$ have the same specializations, then they are rationally equivalent.

(2) Then we prove that if a zero cycle $Z_{k} \in X_{k}$ is rationally equivalent to 0 , then it has a lifting $Z_{K}$ which is also rationally equivalent to 0 .

In order to see (18.1), write

$$
Z_{K}:=Z_{K}^{1}-Z_{K}^{2}=\sum_{j} m_{j}\left[P_{j}\right]
$$

as a sum of irreducible zero cycles. For a point $p \in X_{k}$ let

$$
Z_{K}(p)=\sum_{j}^{(p)} m_{j}\left[P_{j}\right]
$$

be the sum of those points whose specialization is $p$. It is enough to prove that $Z_{K}(p)$ is rationally equivalent to 0 for every $p$.

The next argument follows a suggestion of Colliot-Thélène.

The specialization of $Z_{K}(p)$ is 0 , thus $\sum_{j}^{(p)} m_{j} \operatorname{deg}\left[P_{j}\right]=0$. Let $P \in X_{K}$ be a lifting of $p$. It is then sufficient to prove that

$$
\left[P_{j}\right]-\frac{\operatorname{deg}\left[P_{j}\right]}{\operatorname{deg}[P]}[P]
$$

is rationally equivalent to 0 for every $P_{j}$ specializing to $p$.

Take a base field extension from $K$ to $K\left(P_{j}\right)$. From (19) we see that $K(P) \subset K\left(P_{j}\right)$ thus both $P_{j}$ and $P$ become $K\left(P_{j}\right)$-points which specialize to the same point of $X_{k(p)}$. By (2.1) these two points are R-equivalent, thus $\left[P_{j}\right]-[P] \in \mathrm{CH}_{0}\left(X_{K\left(P_{j}\right)}\right)$ is zero. Its push forward to $X_{K}$ is $\left[P_{j}\right]-$ $\left(\operatorname{deg}\left[P_{j}\right] / \operatorname{deg}[P]\right)[P]$, which is thus also zero in $\mathrm{CH}_{0}\left(X_{K}\right)$.

The proof of (18.2) uses (16).

By assumption, there is a smooth curve and morphisms $f: C \rightarrow \mathbb{P}^{1}$ and $g: C \rightarrow X_{k}$ such that $Z_{k}=g_{*}\left(f^{-1}(0: 1)\right)-g_{*}\left(f^{-1}(1: 0)\right)$.

If $f$ is not separable, then we can factor it as a separable map $f_{1}: C \rightarrow \mathbb{P}^{1}$ composed with a purely inseparable map $\phi: \mathbb{P}^{1} \rightarrow \mathbb{P}^{1}$ (this is always possible for curves over a perfect field). This shows that

$$
Z_{k}=\operatorname{deg} \phi \cdot\left[g_{*}\left(f_{1}^{-1}(0: 1)\right)-g_{*}\left(f_{1}^{-1}(1: 0)\right)\right],
$$

thus it is sufficient to consider the case when $f$ is separable. 
Choose an embedding $j: C \hookrightarrow \mathbb{P}^{m}$ and apply (16) to the morphism

$$
X \times \mathbb{P}^{m} \times \mathbb{P}^{1} \rightarrow \mathbb{P}^{1} \quad \text { with } \quad C^{\prime}:=\operatorname{im}(g \times j \times f) .
$$

There is a comb $C^{*}$ with handle $C^{\prime}$ such that $C^{*}$ has no teeth above the points (1:0), (0:1) $\in \mathbb{P}^{1}$ and $C^{*}$ is unobstructed in the Hilbert scheme as in (17). This gives a curve $C_{K}^{*} \subset X_{K} \times \mathbb{P}^{m} \times \mathbb{P}^{1}$ with projections $F: C_{K}^{*} \rightarrow \mathbb{P}_{K}^{1}$ and $G: C_{K}^{*} \rightarrow X_{K}$. By our construction the degree of $F: C^{*} \rightarrow \mathbb{P}^{1}$ is the same as the degree of $f: C \rightarrow \mathbb{P}^{1}$, thus

$$
Z_{K}:=G_{*}\left(F^{-1}(0: 1)\right)-G_{*}\left(F^{-1}(1: 0)\right)
$$

is a zero cycle on $X_{K}$ which is rationally equivalent to zero and whose specialization is $Z_{k}$.

Lemma 19. Let $(R, m)$ be a Henselian local ring.

(1) There is a one-to-one correspondence between finite étale $R$-algebras and finite separable $R / m$-algebras.

(2) Let $S$ be a finite, flat $R$-algebra such that $S / \sqrt{m S}$ is separable over $R / m$. Then there is a finite étale $R$-algebra $S^{\prime} \subset S$ such that $S / \sqrt{m S} \cong S^{\prime} / \sqrt{m S^{\prime}}$.

Proof. If $(R, m)$ is complete, this is essentially the theory of Witt rings as explained in [Serre62, Chap.II].

In the general case, for the first part see [Milne80, I.4.4]. To see the second part, let $S^{\prime}$ be the finite étale $R$-algebra corresponding to $S / \sqrt{m S}$. Consider $S^{\prime} \otimes_{R} S$. This is a finite, étale $S$-algebra and $S$ is also Henselian by [Milne80, I.4.3]. $S / \sqrt{m S}$ is a direct summand of $S / \sqrt{m S} \otimes_{R / m} S / \sqrt{m S}$, thus by applying the first part to $S^{\prime} \otimes_{R} S$ over $S$ we obtain that $S$ is a direct summand of $S^{\prime} \otimes_{R} S$. This gives the required embedding $S^{\prime} \hookrightarrow S$.

\section{$\S 3 . \quad$ R-equivalence in Families}

Definition 20. A local field $K$ is the quotient field of a complete local Dedekind ring with finite residue field. These are the finite extensions of the $p$-adic fields $\mathbb{Q}_{p}$ and the Laurent series fields $\mathbb{F}_{q}((t))$ over finite fields $\mathbb{F}_{q}$.

The valuation determined by the unique maximal ideal gives a locally compact metric on $K^{n}$. We call this the $p$-adic metric (though for $\mathbb{F}_{q}((t))$ the $t$-adic metric would be a more appropriate name). If $Z$ is a variety over $K$, its $K$-points inherit a well defined topology from its affine pieces, called the $p$-adic topology. $Z(K)$ is locally compact and if $Z$ is projective then $Z(K)$ is compact. 
21 (Proof of (3)). The assertion is local on $Y(K)$, so we may fix a point $0 \in Y(K)$ and prove upper semi continuity there. In the process we are allowed to replace $Y$ with any other $\left(0 \in Y^{\prime}\right) \rightarrow(0 \in Y)$ which is étale at 0 . (This can be done since $Y^{\prime}(K) \rightarrow Y(K)$ is a local homeomorphism in the $p$-adic topology by the inverse function theorem.)

The proof proceeds in 2 steps. First we find finitely many easy to handle open subsets $W_{i} \subset X_{0}(K)$ such that each $W_{i}$ is contained in a single R-equivalence class and the $W_{i}$ lift to nearby fibers $X_{y}$. We are done if the $W_{i}$ are precisely the R-equivalence classes.

If not, then there are finitely many other direct R-equivalences

$$
W_{i} \ni u_{i} \sim u_{j} \in W_{j}
$$

between points of different open sets which generate the full R-equivalence relation. We prove that each of these lifts to every fiber over a suitable $p$-adic neighborhood of $0 \in U_{i j} \subset Y(K)$. Thus over any point of the intersection $\cap U_{i j}$ the number of R-equivalence classes is at most as big as over 0 .

By [Kollár99], for any point $x \in X_{0}(K)$ there is a morphism $g_{x}: \mathbb{P}^{1} \rightarrow X_{0}$ over $K$ such that

(1) $g_{x}(0: 1)=x$,

(2) $g_{x}(1: 0)=: x^{\prime} \neq x$, and

(3) $g_{x}^{*} T_{X_{0}}(-2)$ is ample.

Consider the space of morphisms with universal map

$$
u: \operatorname{Hom}\left(\mathbb{P}^{1}, X_{0},(1: 0) \mapsto x^{\prime}\right) \times \mathbb{P}^{1} \rightarrow X_{0} .
$$

By [Kollár96, II.3.5], there is a neighborhood

$$
\left[g_{x}\right] \in V_{x, 0} \subset \operatorname{Hom}\left(\mathbb{P}^{1}, X_{0},(1: 0) \mapsto x^{\prime}\right)
$$

such that the evaluation map

$$
u^{(0: 1)}: V_{x, 0} \rightarrow X_{0}, \quad \text { given by } \quad v \mapsto u(v,(0: 1))
$$

is smooth. Smooth maps are open in the $p$-adic topology, hence

$$
W_{x, 0}:=u^{(0: 1)}\left(V_{x, 0}(K)\right) \subset X_{0}(K)
$$

is $p$-adic open for every $x$. Since $X_{0}(K)$ is compact, finitely many of these cover $X_{0}(K)$. Let these correspond to the points $x_{1}, \ldots, x_{d}$ and $x_{1}^{\prime}, \ldots, x_{d}^{\prime}$. Note that 
every point in $W_{x_{i}, 0}$ is directly R-equivalent to $x_{i}^{\prime}$, thus in fact any two points in $W_{x_{i}, 0}$ are R-equivalent.

After an étale base change we can assume that there are sections $s_{i}: Y \rightarrow$ $X$ such that $s_{i}(0)=x_{i}^{\prime}$.

Look at the relative space of morphisms with universal map

$$
u: \operatorname{Hom}_{Y}\left(\mathbb{P}^{1}, X,(1: 0) \times Y \mapsto s_{i}(Y)\right) \times \mathbb{P}^{1} \rightarrow X .
$$

As before, for every $i$ there are neighborhoods

$$
\left[g_{x_{i}}\right] \in V_{x_{i}} \subset \operatorname{Hom}_{Y}\left(\mathbb{P}^{1}, X,(1: 0) \times Y \mapsto s_{i}(Y)\right)
$$

such that the evaluation map

$$
u: V_{x_{i}} \rightarrow X, \quad \text { given by } \quad v \mapsto u(v,(0: 1))
$$

is smooth in every fiber and $V_{x_{i}} \cap \operatorname{Hom}_{Y}\left(\mathbb{P}^{1}, X,(1: 0) \times Y \mapsto x_{i}^{\prime}\right)=V_{x_{i}, 0}$.

Set $W_{x_{i}}:=u\left(V_{x_{i}}(K)\right) \subset X(K)$. For every $y$, the intersection $W_{x_{i}} \cap X_{y}(K)$ is a $p$-adic open set and every point of it is directly equivalent to $s_{i}(y)$. Thus $W_{x_{i}} \cap X_{y}(K)$ is contained in a single R-equivalence class. By construction $\cup_{i} W_{x_{i}} \supset X_{0}(K)$, hence there is a $p$-adic neighborhood $0 \in U_{1} \subset Y(K)$ such that

$$
\cup_{i} W_{x_{i}} \supset X_{y}(K) \text { for every } y \in U_{1} \text {. }
$$

This accomplishes the first part of the proof.

Let now $h: \mathbb{P}^{1} \rightarrow X_{0}$ be any morphism and set $u:=h(0: 1)$ and $v:=h(1: 0)$. After an étale base change we can assume that there are sections $\sigma_{u}, \sigma_{v}: Y \rightarrow X$ such that $\sigma_{u}(0)=u$ and $\sigma_{v}(0)=v$.

Let $Z_{u, v, n}$ denote the blow up of $X \times \mathbb{P}^{n}$ along

$$
\sigma_{u}(Y) \times(0: \cdots: 0: 1) \cup \sigma_{v}(Y) \times(1: 0: \cdots: 0)
$$

and $E_{u}, E_{v} \subset Z_{u, v, n}$ the exceptional divisors. As in (17) there is an $n \geq 1$ and a genus 0 comb $C^{*} \subset Z_{u, v, n}$ such that $C^{*}$ intersects the central fibers of $E_{u}, E_{v}$ in a single point transversally and that $\left[C^{*}\right]$ is a smooth point of the relative Hilbert $\operatorname{scheme} \operatorname{Hilb}\left(Z_{u, v, n} / Y\right)$. As before, this implies that the points $\sigma_{u}(y), \sigma_{v}(y) \in X_{y}(K)$ are R-equivalent for every $y$ in a suitable $p$-adic neighborhood of 0 .

This proves the second part and thereby also (3). 


\section{$\S 4 . \quad$ R-equivalence over Large Fields}

The difference between R-equivalence and direct R-equivalence is a frequent source of technical problems. The aim of this section is to prove that these two notions coincide over large fields. A similar result for torsors over finite group schemes is proved in [Moret-Bailly03].

Definition 22. A field $K$ is called large if every $K$-variety with a smooth $K$-point has a dense set of $K$-points.

The best known examples are local fields and infinite algebraic extensions of finite fields.

Theorem 23. Let $K$ be a large field and $X$ a smooth, projective, SRC variety over $K$ with $\operatorname{dim} X \geq 3$. For any set of points $x_{1}, \ldots, x_{n} \in X(K)$ the following two assertions are equivalent:

(1) There is a smooth rational curve $C \subset X$ containing all the points $x_{i}$ such that $T_{X} \otimes \mathcal{O}_{C}(-n)$ is ample.

(2) Any two of the points are R-equivalent.

Proof. It is clear that (23.1) implies (23.2), the interesting part is the converse.

Let us first study the situation for two directly R-equivalent points $x, y \in$ $X(K)$. The direct R-equivalence is given by some morphism $f: \mathbb{P}^{1} \rightarrow X$ with $f(0: 1)=x, f(1: 0)=y$. Consider the comb $C^{*} \subset X \times \mathbb{P}^{n}$ constructed in (15). As in (17) we blow up the points corresponding to $x, y$ and look at the irreducible component $H\left(C^{*}\right)$ of the Hilbert scheme of $B_{x y}\left(X \times \mathbb{P}^{n}\right)$ containing the birational transform of $C^{*}$. By general results on the Hilbert scheme (see, for instance, [Araujo-Kollár03, 37]) $\left[C^{*}\right]$ is a smooth $K$-point of $H\left(C^{*}\right)$ and a general point of $H\left(C^{*}\right)$ corresponds to a smooth rational curve with nef normal bundle. Since $K$ is a large field, $K$-points are dense in $H\left(C^{*}\right)$, hence there is a smooth rational curve $A \subset B_{x y}\left(X \times \mathbb{P}^{n}\right)$ defined over $K$ with nef normal bundle. $A$ intersects each exceptional divisor in a single point, so $A \cong \mathbb{P}^{1}$. Let $g: \mathbb{P}^{1} \cong A \rightarrow X$ be the projection. Undoing the 2 blow ups twists the normal bundle by $\mathcal{O}(2)$, thus, after suitable reparametrization, $g(0: 1)=x, g(1: 0)=y$ and $g^{*} T_{X}$ is ample.

This seems a relatively small advance, but the deformation theory of $g$ is much better than for $f$.

Assume now that we have 3 points $x, y, z$ and $x, y$ and $y, z$ are directly R-equivalent. By the above arguments, these R-equivalences can be realized by 
morphisms $g, h: \mathbb{P}^{1} \rightarrow X$ such that $g(0: 1)=x, g(1: 0)=y, h(0: 1)=y, h(1: 0)=$ $z$ and $g^{*} T_{X}, h^{*} T_{X}$ are ample. The gluing technique (cf. [Kollár96, II.7] or [Araujo-Kollár03, 17]) now implies that there is a $K$-morphism $f_{x z}: \mathbb{P}^{1} \rightarrow X$ such that $f_{x z}(0: 1)=x, f_{x z}(1: 0)=y$ and $f_{x z}^{*} T_{X}$ is ample. By iterating this procedure we obtain that if two points $x, y \in X(K)$ are R-equivalent then there is a $K$-morphism $f_{x y}: \mathbb{P}^{1} \rightarrow X$ such that $f_{x y}(0: 1)=x, f_{x y}(1: 0)=y$ and $f_{x y}^{*} T_{X}$ is ample.

Given our points $x_{1}, \ldots, x_{n} \in X(K)$ choose another point $y$ which is Requivalent to them. (We have at least one rational curve in $X$ through any point, so there are infinitely many points in every R-equivalence class.) There are $K$-morphisms $f_{i}: \mathbb{P}^{1} \rightarrow X$ such that $f_{i}(0: 1)=x_{i}, f_{i}(1: 0)=y$ and $f_{i}^{*} T_{X}$ is ample.

We can now proceed as in [Araujo-Kollár03, 43]. Out of the morphisms $f_{i}$ we assemble a comb $C=C_{0} \cup \cdots \cup C_{n}$ with $C_{i} \cong \mathbb{P}^{1}$ and a morphism $F: C \rightarrow X$ such that $F$ maps $C_{0}$ to $y$ and $\left.F\right|_{C_{i}}=f_{i}$. In [Araujo-Kollár03, 43] we only assumed that the bundles $f_{i}^{*} T_{X}$ are nef, and here they are even ample. By [Araujo-Kollár03, 42] this implies that deformations of $(C, F)$ give morphisms $f: \mathbb{P}^{1} \rightarrow X$ passing through all the points $x_{i}$ and such that $f^{*} T_{X}(-n)$ is ample.

If $\operatorname{dim} X \geq 3$ then we can even find such an $f$ which is an embedding by [Kollár96, II.3.14].

\section{$\S 5$. R-equivalence on Quartics}

It is conjectured that if $K$ is finitely generated over $\mathbb{Q}$ and $X$ is a $K$-variety then $\mathrm{CH}_{0}(X)$ is finitely generated. This would imply that if $X$ is also SRC then $\mathrm{CH}_{0}(X)$ is $\mathbb{Z}+$ (finite group). (See [Colliot-Thélène95] for an overview of the various conjectures and results along these lines.)

This suggests that under similar conditions, $X(K) / R$ might be finite. The aim of the next example is to prove that this is not so, at least over fields of transcendence degree at least 1 . It is still possible that if $K$ is a number field and $X$ is rationally connected then $X(K) / R$ is finite. This is open even for surfaces.

Example 24. Let $Y / \mathbb{Q}$ be any smooth variety. By an observation of [Mumford70], there is an embedding $Y \subset \mathbb{P}^{n}$ such that $Y$ is defined by quadratic equations

$$
Y:=\left(q_{1}=\cdots=q_{m}=0\right)
$$

and $Y$ is contained in a smooth quartic hypersurface $(g=0)$. Consider the 
hypersurface

$$
X_{\mathbb{Q}[t]}:=\left(q_{1}^{2}+\cdots+q_{m}^{2}+t g=0\right) \subset \mathbb{P}_{\text {Spec } \mathbb{Q}[t]}^{n} .
$$

$X_{\mathbb{Q}[t]}$ is a quartic hypersurface. The generic fiber $X_{\mathbb{Q}(t)}$ is smooth and the special fiber is a singular quartic

$$
X_{\mathbb{Q}}=\left(q_{1}^{2}+\cdots+q_{m}^{2}=0\right) .
$$

By our construction, the real points of $X_{\mathbb{Q}}$ coincide with the real points of $Y$, thus the same holds for $\mathbb{Q}$-points:

$$
X_{\mathbb{Q}}(\mathbb{Q})=Y(\mathbb{Q})
$$

Claim 25. There is a surjective specialization map

$$
X_{\mathbb{Q}(t)}(\mathbb{Q}(t)) / R \rightarrow Y(\mathbb{Q}) / R
$$

Proof. The specialization of a $\mathbb{Q}(t)$-point of $X_{\mathbb{Q}(t)}$ is a $\mathbb{Q}$-point of $X_{\mathbb{Q}}$, but these are all in $Y(\mathbb{Q})$. Conversely, if $\left(a_{0}: \cdots: a_{n}\right)$ is a $\mathbb{Q}$-point of $Y(\mathbb{Q})$ then this also gives a point of $X_{\mathbb{Q}[t]}$ since both $g$ and the $q_{i}$ vanish on $Y$. Thus we have a surjective specialization map

$$
X_{\mathbb{Q}(t)}(\mathbb{Q}(t)) / R \rightarrow X_{\mathbb{Q}}(\mathbb{Q}) / R .
$$

The injection $Y \hookrightarrow X_{\mathbb{Q}}$ gives a map

$$
Y(\mathbb{Q}) / R \rightarrow X_{\mathbb{Q}}(\mathbb{Q}) / R
$$

which is surjective. In order to establish our claim, we still need to establish that two $\mathbb{Q}$-points of $Y$ are R-equivalent in $X_{\mathbb{Q}}$ iff they are R-equivalent in $Y$.

To see this, let $h: \mathbb{P}^{1} \rightarrow X_{\mathbb{Q}}$ be any morphism. $\mathbb{Q}$-points of $\mathbb{P}^{1}$ are Zariski dense, thus $h\left(\mathbb{P}^{1}\right)$ is contained in the Zariski closure of $X_{\mathbb{Q}}(\mathbb{Q})$. This is, however, contained in $Y$. Thus any chain of rational curves in $X_{\mathbb{Q}}$ is entirely inside $Y$.

26 (Proof of (5)). With the above notation, let us consider the case when $Y=E \cup Z$ is the disjoint union of an elliptic curve over $\mathbb{Q}$ and another variety $Z$. There are no non-constant maps $\mathbb{P}^{1} \rightarrow E$, thus the above construction gives a quartic hypersurface $H:=X_{\mathbb{Q}(t)}$ with a surjection

$$
X_{\mathbb{Q}(t)}(\mathbb{Q}(t)) / R \rightarrow E(\mathbb{Q})+Z(\mathbb{Q}) / R
$$

If we choose $E$ to have infinitely many $\mathbb{Q}$-points then $X_{\mathbb{Q}(t)}$ has infinitely many R-equivalence classes. 
In order to get a unirational example, choose any elliptic curve over $\mathbb{Q}$ with infinitely many $\mathbb{Q}$-points. We embed $E \hookrightarrow \mathbb{P}^{m}$ by any complete linear system of degree $m+1 \geq 4$. Then $E$ is an intersection of quadrics. (For $m=3$ it is a complete intersection of 2 quadrics, this case is enough for us.) Let $x_{0}, \ldots, x_{m}$ be coordinates on $\mathbb{P}^{m}$ and $q_{r}=0$ the equations of $E$.

Consider now $\mathbb{P}^{m+s+1}$ with coordinates $x_{0}, \ldots, x_{m}, y_{0}, \ldots, y_{s}$ and the equations $q_{r}\left(x_{0}, \ldots, x_{m}\right)$ and $x_{i} y_{j}=0$ for every $i, j$. These equations define a scheme $Y$ with 2 irreducible components. One is $E$ embedded in the linear space ( $y_{0}=$ $\left.\cdots=y_{s}=0\right)$ and the other is the linear space $L=\left(x_{0}=\cdots=x_{m}=0\right) \cong \mathbb{P}^{s}$. $Y=E \cup L$ is an intersection of quadrics and it is contained in a smooth quartic if $s \leq m$.

We can construct $X_{\mathbb{Q}(t)} \subset \mathbb{P}^{m+s+1}$ as above. $X_{\mathbb{Q}(t)}$ has infinitely many R-equivalence classes and it contains a linear space of dimension $s$.

Any smooth quartic of dimension at least 3 is Fano and rationally connected. Unirationality of quartics containing a 2-plane is outlined in [Kollár96, V.5.18]. This gives a degree 6 map $\mathbb{P}^{m+s} \rightarrow X_{\mathbb{Q}(t)}$.

Thus for every $n \geq 5$ we obtain a unirational quartic of dimension $n$ with infinitely many R-equivalence classes.

The Chow group of this example does not seem to be very interesting. Every degree zero 0-cycle is 6-torsion and we only get a surjection from $\mathrm{CH}_{0}^{0}(H)$ to a torsion quotient of $E(\mathbb{Q})$ and the latter is finite. The relevant quotient is probably $E(\mathbb{Q}) / 2 E(\mathbb{Q})$.

Remark 27. One can get similar examples where $X$ is a complete intersection of 3 quadrics. Indeed, start with 2 quadrics in 4 variables $q_{1}\left(x_{0}, \ldots, x_{4}\right)$, $q_{2}\left(x_{0}, \ldots, x_{4}\right)$ whose intersection is a smooth elliptic curve $E \subset \mathbb{P}_{\mathbb{Q}}^{3}$. Let $Q_{i}$ for $i=1,2,3$ be quadrics in the variables $x_{0}, \ldots, x_{n}$ such that $Q_{1}=Q_{2}=Q_{3}$ is smooth and contains $E$. Consider

$$
X_{\mathbb{Q}[t]}:=\left(q_{1}+t Q_{1}=q_{2}+t Q_{2}=x_{5}^{2}+\cdots x_{n}^{2}+t Q_{3}=0\right) \subset \mathbb{P}_{\mathbb{Q}[t]}^{n} .
$$

As before we obtain that there are infinitely many R-equivalence classes on $X_{\mathbb{Q}(t)}$ if $E$ has infinitely many $\mathbb{Q}$-points.

28 (Proof of $(6)$ ). Let $k / \mathbb{Q}$ be a field extension of degree $d$. Construct a quartic $X_{d}$ over $\mathbb{Q}(t)$ using $Y=\operatorname{Spec} k$ as in (24). $X_{d}$ has a point over $k(t)$ and $\operatorname{deg} k(t) / \mathbb{Q}(t)=d$.

Assume that $X_{d}$ has a point $P$ over a field extension $L^{\prime} / \mathbb{Q}(t)$ of odd degree $d^{\prime}$. Specialize this point to a zero cycle $\sum m_{i}\left[p_{i}\right]$ of degree $d^{\prime}$ on $Y_{d}$. One of the 
$p_{i}$, say $p_{1}$, has odd degree. $p_{1} \notin X_{\mathbb{Q}} \backslash Y_{\mathbb{Q}}$ since otherwise we would obtain a point of the quadric $u_{1}^{2}+\cdots+u_{m}^{2}=0$ over an odd degree extension. Thus $p_{1}$ is contained in $Y=\operatorname{Spec} k$, hence its degree is divisible by $d$. Thus $d^{\prime} \geq d$.

\section{Acknowledgements}

I thank C. Araujo, J.-L. Colliot-Thélène and E. Szabó for helpful comments. This paper was completed during my stay at the Isaac Newton Institute for Mathematical Sciences, Cambridge. Partial financial support was provided by the NSF under grant numbers DMS-9970855 and DMS02-00883.

\section{References}

[Araujo-Kollár03] Araujo, C. and Kollár, J., Rational curves on varieties, in: Higher dimensional varieties and rational points, Bolyai Soc. Math. Stud., 12 (2003), 13-68.

[Artin69] Artin, M., Algebraization of formal moduli. I, 1969 Global Analysis (Papers in Honor of K. Kodaira) pp. 21-71 Univ. Tokyo Press, Tokyo.

[Colliot-Thélène83] Colliot-Thélène, J.-L., Hilbert's Theorem 90 for $K_{2}$, with application to the Chow groups of rational surfaces, Invent. Math., 71 (1983), 1-20.

[Colliot-Thélène95] _ L L'arithmétique du groupe de Chow des zéro-cycles. Les Dixhuitimes Journées Arithmétiques (Bordeaux, 1993), J. Théor. Nombres Bordeaux, 7 (1995), 51-73.

[Colliot-Thélène00] line, Ann. of Math., 151 (2000), 359-373.

[Coray76] Coray, D., Algebraic points on cubic hypersurfaces, Acta Arith., 30 (1976), 267-296.

[Fulton84] Fulton, W., Intersection theory, Springer Verlag, 1984.

[Gr-Ha-St03] Graber, T., Harris, J. and Starr, J., Families of rationally connected varieties, J. Amer. Math. Soc., 16 (2003), 57-67.

[Kollár96] Kollár, J., Rational Curves on Algebraic Varieties, Springer, 1996.

[Kollár98] _ L Low degree polynomial equations: arithmetic, geometry and topology. European Congress of Mathematics, Vol. I (Budapest, 1996) 255-288, Progr. Math., 168, Birkhäuser, Basel, 1998.

[Kollár99] _ Rationally connected varieties over local fields, Ann. of Math., 150 (1999), 357-367.

[Kollár01] _ Which are the simplest Algebraic Varieties?, Bull. AMS, 38 (2001), 409-433.

[Ko-Mi-Mo92] Kollár, J., Miyaoka, Y. and Mori, S., Rationally Connected Varieties, J. Alg. Geom., 1 (1992), 429-448.

[Kollár-Szabó03] Kollár, J. and Szabó, E., Rationally connected varieties over finite fields, Duke Math. J., 120 (2003), 251-267.

[Madore03] Madore, D., Équivalence rationnell sur les hypersurfaces cubiques sur les corps p-adiques, Manuscr. Math., 110 (2003), 171-185.

[Manin72] Manin, Yu. I., Cubic forms, Nauka, 1972.

[Milne80] Milne, J., Étale cohomology, Princeton Univ. Press, 1980.

[Moret-Bailly01] Moret-Bailly, L., R-équivalence simultanée de torseurs: un complément á l'article de P. Gille, J. Number Theory, 91 (2001), 293-296. 
[Moret-Bailly03] Moret-Bailly, L., Sur la R-equivalence de torseurs sous un groupe fini, J. Number Theory, 99 (2003), 383-404.

[Mumford70] Mumford, D., Varieties defined by quadratic equations, Questions on Algebraic Varieties (C.I.M.E., III Ciclo, Varenna, 1969) 29-100. Edizioni Cremonese, Rome, 1970.

[Serre62] Serre, J.-P., Corps locaux, Hermann, 1962.

[SGA1] Grothendieck, A., Revêtements étales et groupes fondamentale, Springer Lecture Notes, 224 (1971). 